\title{
HDAC inhibition activates the apoptosome via Apaf1 upregulation in hepatocellular carcinoma
}

\author{
Reena Buurman, Maria Sandbothe, Brigitte Schlegelberger and Britta Skawran*
}

\begin{abstract}
Background: Histone deacetylation, a common hallmark in malignant tumors, strongly alters the transcription of genes involved in the control of proliferation, cell survival, differentiation and genetic stability. We have previously shown that HDAC1, HDAC2, and HDAC3 (HDAC1-3) genes encoding histone deacetylases 1-3 are upregulated in primary human hepatocellular carcinoma $(\mathrm{HCC})$. The aim of this study was to characterize the functional effects of HDAC1-3 downregulation and to identify functionally important target genes of histone deacetylation in HCC.

Methods: Therefore, HCC cell lines were treated with the histone deacetylase inhibitor (HDACi) trichostatin A and by siRNA-knockdown of HDAC1-3. Differentially expressed mRNAs were identified after siRNA-knockdown of HDAC1-3 using mRNA expression profiling. Findings were validated after siRNA-mediated silencing of HDAC1-3 using qRTPCR and Western blotting assays.

Results: mRNA profiling identified apoptotic protease-activating factor 1 (Apaf1) to be significantly upregulated after HDAC inhibition (HLE siRNA\#1/siRNA\#2 $p<0.05$, HLF siRNA\#1/siRNA\#2 $p<0.05$ ). As a component of the apoptosome, a caspase-activating complex, Apaf1 plays a central role in the mitochondrial caspase activation pathway of apoptosis. Using annexin $\mathrm{V}$, a significant increase in apoptosis could also be shown in HLE (siRNA \#1 $p=0.0034$ ) and HLF after siRNA against HDAC1-3 (Fig. 3a, b). In parallel, caspase-9 activity was increased after siRNA-knockdown of HDAC1-3 leading to enhanced apoptosis after HDAC inhibition (Fig. 3c, d).
\end{abstract}

Conclusions: The present data show that siRNA-knockdown of HDAC1-3 plays a major role in mediating apoptotic response to HDAC inhibitors through regulation of Apaf1.

Keywords: Hepatocellular carcinoma, Acetylation, Histone deacetylases, Apoptosis, Trichostatin A

\section{Background}

Hepatocellular carcinoma (HCC) represents the fourth most common malignant tumor with more than 1 million persons affected per year worldwide $[1,2]$. HCC is associated with a very poor prognosis (http://www-dep.iarc. $\mathrm{fr} / \mathrm{f}[3,4]$ and only limited treatment options are available. Therefore, new effective therapeutic strategies are urgently needed.

Currently, cancer development is regarded as an interaction of genetic, genomic and epigenetic alterations

\footnotetext{
*Correspondence: Skawran.britta@mh-hannover.de Institute of Human Genetics, Hannover Medical School, Carl-Neuberg-Straße 1, 30625 Hannover, Germany
}

[5-9]. The histone modification, i.e. acetylation, of lysine residues is an important secondary modification responsible for chromatin remodeling and is controlled by histone acetyltransferases (HATs) and histone deacetylases (HDACs). The deacetylation of chromatin results in the formation of a higher order, i.e. condensation of chromatin, leading to the repression of transcription of many genes involved in multiple cellular functions. Notably, epigenetic modifications are reversible and can be targeted by new drugs like DNA methyltransferase and HDAC inhibitor (HDACi), drugs that have shown efficacy in clinical phase I/II studies (http://www.clinicaltrials.gov) [10]. HDACi are found to have an anti-cancer function in many different tumors $[11,12]$. 
We have previously shown that $H D A C 1-3$ are upregulated in primary human HCC [13]. Therefore, we hypothesized that the altered expression of genes due to chromatin remodeling may play a fundamental role in hepatocarcinogenesis. We hence induced histone acetylation by HDACi or siRNA silencing of $H D A C 1-3$ to identify functionally important target genes. Upon increasing histone acetylation, the apoptotic proteaseactivating factor 1 (Apaf1), a major regulator of apoptosis, was reactivated.

\section{Methods}

\section{Primary tissue}

Analysis was carried out based on the re-evaluation of pseudonymized tumor specimens of 23 patients with HCC treated at Hannover Medical School (MHH) and taken from the archive of the Institute of Pathology at the MHH (Germany) [13]. The local Ethics Committee ("Ethikkommission der Medizinischen Hochschule Hannover", head: Prof. Dr. H.D. Tröger) approved the application to retrospectively use the samples in this study (left over from diagnostic procedures) that had been irreversibly unlinked from the source, rendering them anonymous, and thus exempting them from IRB review, waiving the consent requirement due to no legal or ethical concerns (Ethics Statement: No. 2208-2014).

\section{Cell culture, HDAC inhibition and transfection}

HCC cell lines HLE [14] and HLF [14] (kindly provided by Professor Nam-Ho Huh, Department of Cell Biology, Graduate School of Medicine, Dentistry and Pharmaceutical Sciences, Okayama University, Okayama, Japan) were treated with trichostatin A (TSA) or transfected with siRNA against $H D A C 1-3$ as previously described [13]. siRNA\# 1 and $\# 2$ are different mixtures of siRNAs against HDAC1, HDAC2 and HDAC3 provided by Qiagen, Hilden, Germany (siRNA\#1 = Hs_HDAC1_1, Hs HDAC2_3, Hs_HDAC3_10; siRNA\#2 = Hs_HDAC1_6, Hs_HDAC2_1, Hs_HDAC3_9).

\section{Expression analyses}

Microarray analyses were done as previously described with Whole Human Genome Oligo Microarray Kit $4 \times 44 \mathrm{k}$ (Agilent) [13]. The array analysis was performed by a paired $t$ test of treated against untreated cells with a corrected (Benjamini-Hochberg) $p$ value of 0.1. The Agilent GeneSpring GX Data Analysis Software was used for bioinformatic analysis. mRNAs of interest were validated by qRTPCR as previously described [13] with Taqman Assay [Hs00559421_m1, amplicon spans exon 10 and 11 with a length of 112 bp (Applied Biosystems)] and Western blot using antibody against Apaf1
[\#8723 Cell Signaling/NEB Danvers, MA, USA, Antibody ID: AB_10829610 from http://www.antibodyregistry. org, used 1:1000, blocking with 3 \% Slim Fast chocolate (Allpharm)].

\section{Assays to determine apoptosis and caspase- 9 activity}

To detect apoptotic cells, both adherent and floating cells were collected and washed twice with PBS. Cells were resuspended in $1 \times$ binding buffer (Becton-Dickinson) and stained with $5 \mu \mathrm{L}$ annexinV-APC (Becton-Dickinson) and $5 \mu \mathrm{L}$ 7-AAD (Becton-Dickinson) for $15 \mathrm{~min}$ in the dark. Samples were analyzed with the FACSCalibur flow cytometer (Becton-Dickinson). Data analysis was performed using CellQuest Pro software (Becton-Dickinson).

To detect viable cells, the activity of the mitochondrial dehydrogenase was determined using the Cell Proliferation Reagent WST-1 in 96-well format (Roche, Mannheim, Germany). To determine caspase-9 activity, Caspase-Glo ${ }^{\circledR} 9$ Assay (G8210, Promega) was used. Absorption was measured using the Synergy 2 MultiMode Microplate Reader (BioTek).

\section{Statistics}

For statistical analysis, GraphPad Prism version 5.02 for Windows was used. Each assay (except for microarrays) was performed three times in biologically independent assays. 1-way ANOVA with Dunnett's multiple comparison test was performed. Statistics are given as mean \pm standard deviation. Asterisks are related to the following $p$ values in all experiments: ${ }^{*} p=0.01-0.05$ significant, ${ }^{* *} p=0.001-0.01$ very significant, and $\stackrel{* * * 0}{*} p<0.001$ extremely significant.

\section{Results}

As recently published by Buurman et al. [13], $H D A C 1-3$ are upregulated in human primary $\mathrm{HCC}$. To characterize the functional effects of deregulated $H D A C 1-3, \mathrm{HCC}$ cell lines HLE, HLF, HepG2 and Huh-7 were treated with HDACi TSA. To rule out such unspecific and toxic side effects of HDACs by inhibitors like TSA in the two cell lines HLE and HLF, siRNA-knockdown of combined HDAC1-3 was performed.

Aiming to determine the influence of increased histone acetylation on mRNA expression in HCC, we investigated global mRNA expression by microarray analyses in HCC cell lines treated with specific siRNA against HDAC1-3 for $48 \mathrm{~h}$.

This led to significant differences in gene expression between treated and untreated cells. Apaf1 showed the most pronounced differential expression levels compared to untreated cells. Since Apaf1 is a central protein of the intrinsic apoptotic pathway and the core molecule in the 
a

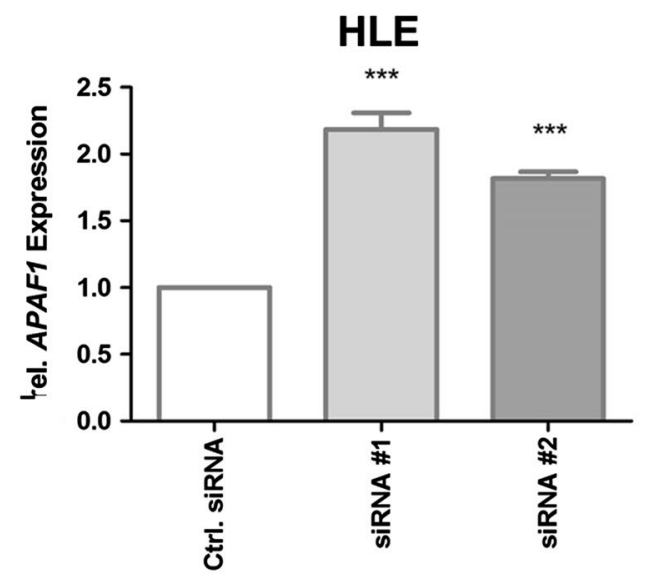

C

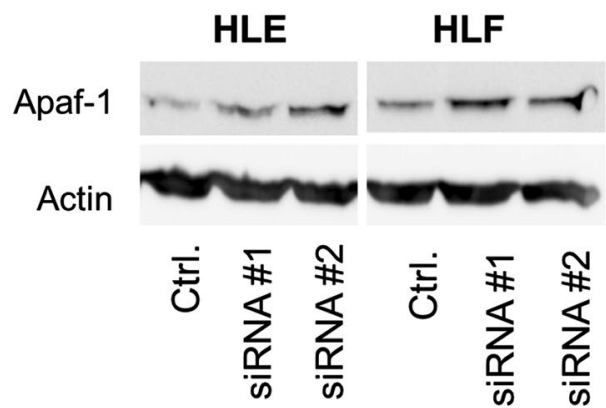

b

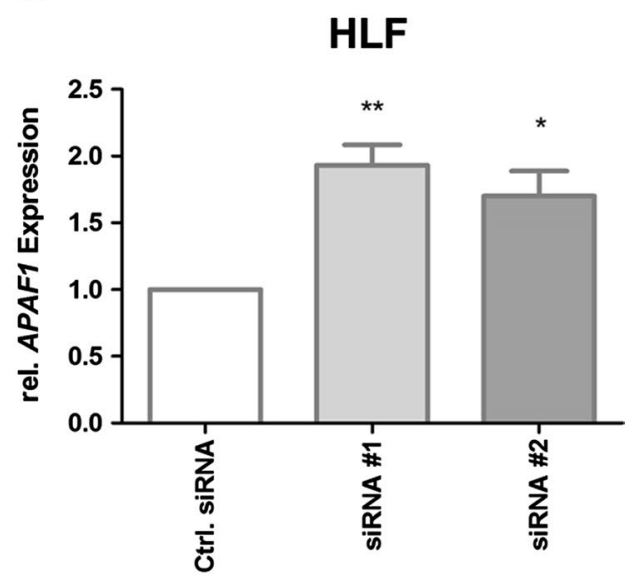

Fig. 1 Expression of Apaf1 after siRNA transfection against HDAC1-3 in HLE and HLF. 48 h after transfection of siRNA against HDAC1-3, the HCC cell lines $\mathbf{a} \mathrm{HLE}$ and $\mathbf{b}$ HLF showed a significant increased expression of Apaf1 measured by qRTPCR. c Western blot analysis of Apaf1 after siRNA showed a significant increase in Apaf1 expression compared with controls. Values are mean \pm SEM, $n=3 .{ }^{* * *} p<0.001,{ }^{* *} p<0.01,{ }^{*} p \leq 0.05$, ANOVA plus Dunnett's posttest

formation of the apoptosome, a caspase-activating complex, this gene was investigated further.

As shown in Fig. 1a, b, qRTPCR confirmed the result of the array analyses and showed a systematic increase in Apaf1 expression after siRNA treatment against $H D A C 1-$ 3 (HLE siRNA\#1/siRNA\#2 $p<0,05$, HLF siRNA\#1/ siRNA\#2 $p<0,05)$. HLE and HLF showed increased Apaf1 expression using siRNA\#1 about 118/93 \% in comparison to the controls and using siRNA\#2 about $81 / 70 \%$. Furthermore, expression of Apaf1 was analyzed by Western blot analyses (Fig. 1c) in HLE and HLF cell lines after siRNA treatment against $H D A C 1-3$. An increase in Apaf1 protein expression was seen after histone deacetylation inhibition, substantiating also the result of the microarray analyses.

Moreover, qRTPCR was performed focusing on Apaf1 in the four HCC cell lines HLE, HLF, HepG2 and Huh-7 which were incubated with HDACi TSA for $12 \mathrm{~h}$ to validate the results from previous experiments. As shown in Fig. 2, qRTPCR confirmed the result of siRNA treatment against $H D A C 1-3$ and showed an increase in Apaf1 expression after HDACi treatment. HLE, Huh7 and HepG2 showed an increased Apaf1 expression with a significance of $p=0.01-0.05$ (HLE $68 \%$, HLF $59 \%$, Huh7 $173 \%$, HepG2 $=124 \%$ increase).

To investigate the effect of histone acetylation and upregulation of Apaf1 on apoptosis induction, apoptosis and proliferation were measured $48 \mathrm{~h}$ after siRNA transfection against $H D A C 1-3$. In a previous study [13], it could be shown that acetylation was increased by more than $50 \%$ and a significant increase in caspase $3 / 7$ activity of more than $50 \%$ than in controls. Using annexin V, a significant increase in apoptosis could also be shown in HLE [siRNA\#1 $80 \%$, siRNA\#2 $20 \%$ increase (siRNA $\# 1 p=0.0034$ )] and HLF (siRNA\#1 $38 \%$, siRNA\#2 $28 \%$ increase) after siRNA against $H D A C 1-3$ (Fig. 3a, b). In parallel, both HCC cell lines showed an increase of caspase 9 activity after $48 \mathrm{~h}$ compared with controls (Fig. 3c, d). The increase was between 26 and $76 \%$ with exception of siRNA\#2 in HLE.

To confirm the clinical relevance of the deregulation of Apaf1, 23 primary HCC were investigated using qRTPCR. 




Fig. 2 Expression of Apaf1 after 12-h treatment with the HDACi TSA. Expression of Apaf1 after 12-h treatment with the HDACi TSA in the HCC cell lines HLE, HLF, Huh7 and HepG2 measured by qRTPCR. After treatment, Apaf1 showed an increased expression compared with controls. Values are mean $\pm \mathrm{SEM}, n=3,{ }^{*} p \leq 0.05$, ANOVA plus Dunnett's posttest
Compared to a human reference, Apaf1 expression was reduced in more than half of the primary HCC (Fig. 4).

\section{Discussion}

Histone deacetylation represents an important epigenetic modification in cancer development and is responsible for condensation of chromatin and transcriptional silencing in the respective genomic regions. In a previous study, we have shown that $H D A C 1-3$ are consistently upregulated in HCC [13].

Transcriptional repressors such as class I HDACs are receiving growing attention as potential therapeutic targets in human malignancies [15]. Several HDACi have been identified that drive tumor cells into growth arrest, differentiation, and apoptotic cell death [16]. Meanwhile several HDAC-specific inhibitors are being tested in preclinical and clinical trials [15].

In a previous study, we have shown that treatment of HCC cell lines with the HDACi TSA or with specific a

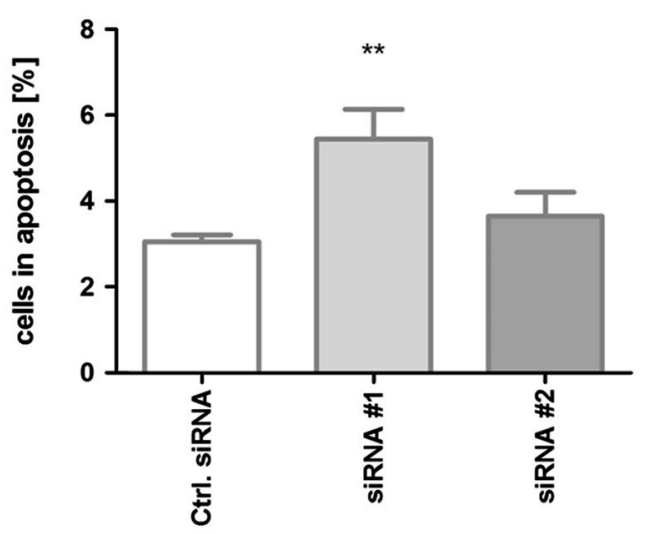

C

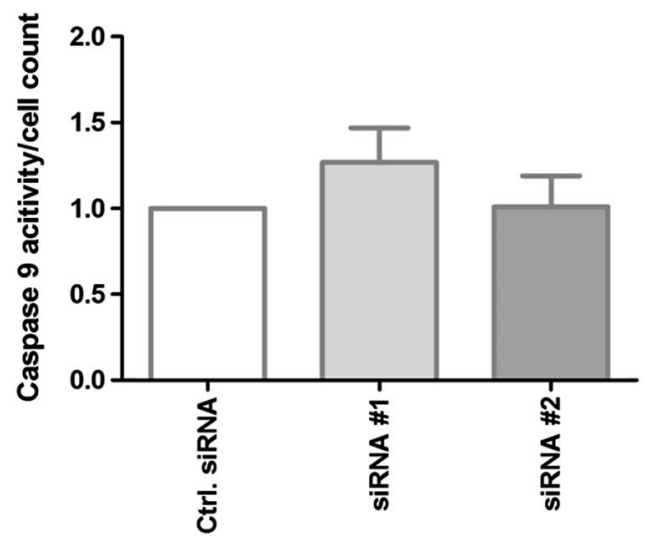

b

HLF

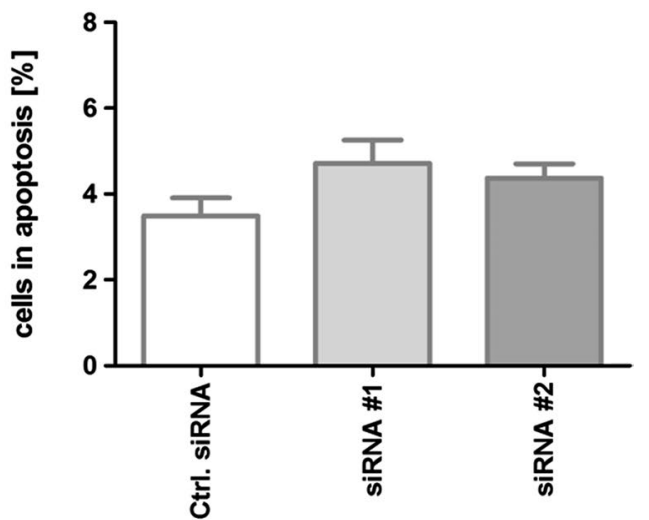

d

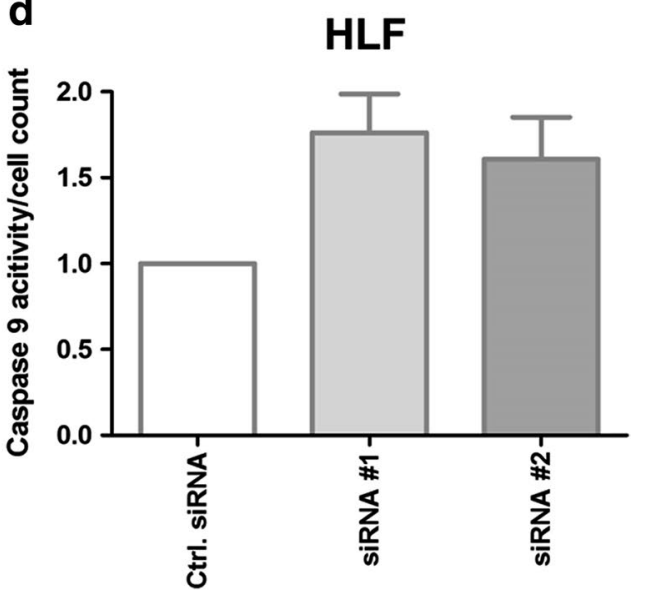

Fig. 3 Apoptosis after siRNA transfection against HDAC1-3. Apoptosis measured by flow cytometry using annexin $\mathrm{V}$ staining in HLE $\mathbf{a}$ and HLF $\mathbf{b}$ is increased in both cell lines. Caspase-9 activity in the HCC cell lines $\mathbf{c}$ HLE and $\mathbf{d}$ HLF normalized to cell count after $48 \mathrm{~h}$ showed a significant increase in caspase- 9 activity in both cell lines compared with controls. Values are mean $\pm \mathrm{SEM}, n=3 .{ }^{* *} p<0.01,{ }^{*} p=<0.05$, ANOVA plus Dunnett's posttest 


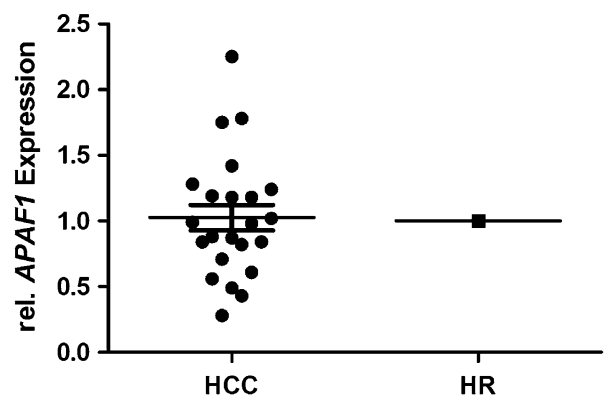

Fig. 4 Expression of APAF1 measured by qRTPCR in 23 primary HCC compared to a human reference. Primary human HCC showed reduced expression of Apaf1 compared to a human reference

siRNA against $H D A C 1,2$ and 3 led to increased histone acetylation [13]. Using gene expression profiling after specific downregulation of $H D A C 1,2$ and 3 in $\mathrm{HCC}$ cell lines HLE and HLF, we observed an upregulation of Apaf1. Apaf1 showed the most pronounced differential expression levels compared to untreated cells. This gene plays a central role in the mitochondrial caspase activation pathway of apoptosis. Indeed, after siRNA treatment against $H D A C 1-3$, Apaf1 expression was increased, caspase- 9 was activated and apoptosis induced significantly [13].

Human cancers are characterized by an imbalance of regulatory mechanisms controlling different cellular pathways, including apoptosis. Apaf1 and the cysteine proteases known as caspases are central proteins of the intrinsic apoptotic pathway. This pathway of apoptosis is activated by death stimuli that induce the release of proapoptotic factors such as cytochrome $c$ from mitochondria into the cytosol and trigger the subsequent formation of the apoptosome, an oligomeric protein complex consisting of Apaf1, procaspase-9, cytochrome $c$, and deoxyadenosine triphosphate (ATP) [17]. Mature caspase-9 activates downstream caspases, such as caspase-3, resulting in the controlled demise of the cell [18]. It is known that the histone acetylation and deacetylation in the retina are epigenetic phenomena that have an influence on the transcriptional repression of Apaf1 [19]. Furthermore, Tan et al. [20] could show that TSA restores Apaf1 function in chemoresistant ovarian cancer cells. It is also known that Apaf1 is inactivated in metastatic melanomas [21]. Interestingly, TSA treatment did not result in increased Apaf1 levels in melanoma cells, suggesting that HDAC inhibitor effects on apoptotic factors may be celltype-specific [22]. Moreover, it is known that HDACi can induce p53 acetylation which leads to the transcriptional upregulation of Apaf1 [23]. Moreover, Hanigan et al. [24] could show that HDAC2 regulates Apaf1 mediating the apoptotic response to HDAC inhibitors.

\section{Conclusions}

Our studies demonstrate that $H D A C 1-3$ play a major role in mediating apoptotic response to HDAC inhibitors through direct regulation of Apaf1 in HCC.

\section{Abbreviations}

Apaf1: apoptotic protease-activating factor 1; HCC: hepatocellular carcinoma; HDAC: histone deacetylases; HDACi: histone deacetylase inhibitor; qRTPCR: quantitative reverse-transcription polymerase chain reaction; siRNA: small interfering RNA; TSA: trichostatin A.

\section{Authors' contributions}

RB acquisition of data, drafting the manuscript. MS acquisition of data. BS study design, revising it critically for important intellectual content. BS study design, drafting the manuscript. All authors read and approved the final manuscript.

\section{Competing interests}

The authors declare that they have no competing interests.

Received: 10 September 2015 Accepted: 11 May 2016

Published online: 24 June 2016

\section{References}

1. Colombo M. Treatment of hepatocellular carcinoma. J Viral Hepat. 1997:4(Suppl 1):125-30.

2. Herold C, ReckT, Fischler P, Ott R, Radespiel-Troeger M, Ganslmayer M, et al. Prognosis of a large cohort of patients with hepatocellular carcinoma in a single European centre. Liver. 2002;22(1):23-8.

3. Mitelman F. Catalog of chromosome aberrations in cancer. vol. 1. New York: Wiley; 1994.

4. Okuda K. Hepatocellular carcinoma. J Hepatol. 2000;32(1 Suppl):225-37

5. Lehmann U, Berg-Ribbe I, Wingen LU, Brakensiek K, Becker T, Klempnauer J, et al. Distinct methylation patterns of benign and malignant liver tumors revealed by quantitative methylation profiling. Clin Cancer Res. 2005;11(10):3654-60.

6. Marks P, Rifkind RA, Richon VM, Breslow R, Miller T, Kelly WK. Histone deacetylases and cancer: causes and therapies. Nat Rev Cancer. 2001;1(3):194-202.

7. Ropero S, Esteller M. The role of histone deacetylases (HDACs) in human cancer. Molecular Oncology. 2007;1 (1):19-25.

8. Schneider-Stock R, Ocker M. Epigenetic therapy in cancer: molecular background and clinical development of histone deacetylase and DNA methyltransferase inhibitors. IDrugs. 2007;10(8):557-61.

9. Fraga MF, Ballestar E, Villar-Garea A, Boix-Chornet M, Espada J, Schotta $\mathrm{G}$, et al. Loss of acetylation at Lys 16 and trimethylation at Lys 20 of histone $\mathrm{H} 4$ is a common hallmark of human cancer. Nat Genet. 2005;37(4):391-400.

10. Hauns B, Mais A, Doblhofer R, Henning SW, Hentsch B, Bitzer M. OP-09 Pharmacokinetic characteristics of the new treatment combination of sorafenib and resminostat, a novel histone deacetylase (HDAC) inhibitor, in patients with advanced hepatocellular carcinoma (HCC): the SHELTER study. Dig Liver Dis. 2013;45(Supplement 3(0)):S251.

11. Dokmanovic M, Marks PA. Prospects: histone deacetylase inhibitors. J Cell Biochem. 2005;96(2):293-304.

12. Walkinshaw DR, Yang XJ. Histone deacetylase inhibitors as novel anticancer therapeutics. Curr Oncol. 2008;15(5):237-43.

13. Buurman R, Gurlevik E, Schaffer V, Eilers M, Sandbothe M, Kreipe H, et al. Histone deacetylases activate hepatocyte growth factor signaling by repressing microRNA-449 in hepatocellular carcinoma cells. Gastroenterology. 2012;143(3):811-20, e1-15.

14. Dor I, Namba M, Sato J. Establishment and some biological characteristics of human hepatoma cell lines. Gan. 1975;66(4):385-92. 
15. Shankar S, Srivastava RK. Histone deacetylase inhibitors: mechanisms and clinical significance in cancer: HDAC inhibitor-induced apoptosis. Adv Exp Med Biol. 2008;615:261-98.

16. Marks PA, Richon VM, Rifkind RA. Histone deacetylase inhibitors: inducers of differentiation or apoptosis of transformed cells. J Natl Cancer Inst. 2000;92(15):1210-6.

17. Fadeel B, Ottosson A, Pervaiz S. Big wheel keeps on turning: apoptosome regulation and its role in chemoresistance. Cell Death Differ. 2008;15(3):443-52.

18. Green DR, Reed JC. Mitochondria and apoptosis. Science. 1998:281(5381):1309-12.

19. Wallace DM, Cotter TG. Histone deacetylase activity in conjunction with E2F-1 and p53 regulates Apaf-1 expression in $661 \mathrm{~W}$ cells and the retina. J Neurosci Res. 2009;87(4):887-905.

20. Tan L, Kwok RP, Shukla A, Kshirsagar M, Zhao L, Opipari AW Jr, et al. Trichostatin A restores Apaf-1 function in chemoresistant ovarian cancer cells. Cancer. 2011;117(4):784-94.
21. Soengas MS, Capodieci P, Polsky D, Mora J, Esteller M, Opitz-Araya X, et al. Inactivation of the apoptosis effector Apaf-1 in malignant melanoma. Nature. 2001;409(6817):207-11.

22. Facchetti F, Previdi S, Ballarini M, Minucci S, Perego P, La Porta CA. Modulation of pro- and anti-apoptotic factors in human melanoma cells exposed to histone deacetylase inhibitors. Apoptosis. 2004;9(5):573-82.

23. Yun T, Yu K, Yang S, Cui Y, Wang Z, Ren H, Chen S, Li L, Liu X, Fang M, Jiang $X$. Acetylation of p53 protein at lysine 120 up-regulates Apaf-1 protein and sensitizes the mitochondrial apoptotic pathway. J Biol Chem. 2016;291(14):7386-95.

24. Hanigan CL, Van Engeland M, De Bruine AP, Wouters KA, Weijenberg $M P$, Eshleman JR, et al. An inactivating mutation in HDAC2 leads to dysregulation of apoptosis mediated by APAF1. Gastroenterology. 2008;135(5):1654-64

\section{Submit your next manuscript to BioMed Central and we will help you at every step:}

- We accept pre-submission inquiries

- Our selector tool helps you to find the most relevant journal

- We provide round the clock customer support

- Convenient online submission

- Thorough peer review

- Inclusion in PubMed and all major indexing services

- Maximum visibility for your research

Submit your manuscript at www.biomedcentral.com/submit 